\title{
Sensitivity Enhancement by Exchange Mediated Magnetization Transfer of the Xenon Biosensor Signal
}

\author{
Sandra Garcia ${ }^{\text {a,c }}$ Lana Chavez ${ }^{\mathrm{a}, \mathrm{c}}$ Thomas J. Lowery ${ }^{\mathrm{b}, \mathrm{c}}$ \\ Song-I Han ${ }^{\mathrm{d}}$ David E. Wemmer ${ }^{\mathrm{b}, \mathrm{c}}$ Alexander Pines ${ }^{\mathrm{a}, \mathrm{c}, *}$ \\ ${ }^{a}$ Materials Science Division, Lawrence Berkeley National Laboratory \\ ${ }^{\mathrm{b}}$ Physical Biosciences Division, Lawrence Berkeley National Laboratory \\ ${ }^{\mathrm{c}}$ Department of Chemistry, University of California, Berkeley 94720 \\ ${ }^{\mathrm{d}}$ Department of Chemistry, University of California, Santa Barbara 93106
}

\begin{abstract}
Hyperpolarized xenon associated with ligand derivitized cryptophane-A cages has been developed as a NMR based biosensor. To optimize the detection sensitivity we describe use of xenon exchange between the caged and bulk dissolved xenon as an effective signal amplifier. This approach, somewhat analogous to 'remote detection' described recently, uses the chemical exchange to repeatedly transfer spectroscopic information from caged to bulk xenon, effectively integrating the caged signal. After an optimized integration period, the signal is read out by observation of the bulk magnetization. The spectrum of the caged xenon is reconstructed through use of a variable evolution period before transfer and Fourier analysis of the bulk signal as a function of the evolution time.
\end{abstract}

Key words: xenon, biosensor, remote detection, chemical exchange

\section{Introduction}

Nuclear Magnetic Resonance is an indispensable tool used in applications as diverse as protein structure determination, the study of porous materials, and medical imaging. NMR's biggest limitation, however, is the low thermal polarization of traditionally studied nuclei that leads to low signal. Hyperpolarized

* Corresponding author

Email address: pines@berkeley.edu (Alexander Pines). 
gases such as xenon have helped to overcome this limitation because they can be generated with 4 to 5 orders of magnitude increase in polarization by optical pumping [1]. Although their use is limited to specific systems, the wealth of information they provide is by other means unattainable. Xenon is particularly useful because it has a large range in chemical shift and because it dissolves readily into hydrophobic pockets of proteins [2-7] and lung tissue [8]. In many cases xenon is also in slow exchange among different sites on the NMR timescale, giving rise to a distinct chemical shift for each site. Xenon biosensors [9] advantageously use the above-mentioned properties. An avidin specific sensor has previously been used to detect the presence of avidin protein at low concentrations [10]. The potential for these sensors as targeted contrast agents for imaging was also recently shown [11].

Xenon biosensors are composed of a xenon-binding cryptophane cage, a solubilizing moeity such as a short peptide, and a targeting group such as a ligand or antibody. The xenon exchange properties of these biosensors have been previously used to improve their detection limit by two orders of magnitude [12]. This was accomplished by utilizing the large pool of water-dissolved xenon as a polarization reservoir from which magnetization can be efficiently transferred to the xenon biosensor. Because the xenon biosensor resonance is well resolved from that of water-dissolved xenon, a selective pulse can be used to acquire the signal of interest without perturbing the polarization reservoir. The relatively rapid exchange of the caged xenon $(\sim 70 \mathrm{~ms})$ with that in water refreshes the polarization of the cryptophane-bound xenon, so that signal acquisition can be quickly repeated. For this type of signal adding, which has been termed Exchange Signal Averaging (ESA), the conventional signal-tonoise ratio $(\mathrm{S} / \mathrm{N})$ increases by $\sqrt{N}$, where $N$ is the number of scans, since both signal and noise are collected in every scan. The pulse sequence for ESA is shown in Figure 1B. Here we describe an alternative exchange transfer approach for xenon biosensor detection that intrinsically scales linearly with the number of 'scans' $N$ rather than $\sqrt{N}$. This offers a further sensitivity gain, particularly under conditions of very dilute sensors.

\section{Materials and Methods}

All spectra were acquired using a $7 \mathrm{~T}$ superconducting magnet from Oxford Instruments, equipped with a Unity Inova spectrometer (Varian Inc., Palo Alto, CA). Hyperpolarized xenon was prepared in a MITI polarizer from Amersham, that produces $\sim 3 \%$ polarization with a gas mixture of 1:10:89, Xe: $\mathrm{N}_{2}: \mathrm{He}$, respectively. Xenon was introduced into the samples using a flow apparatus as described in reference [12]. The biosensor used was composed of cryptophaneA cage linked to biotin via 6 glycine units with an additional peptide that solubilizes the biosensor (structure 3 described in reference [10]). Data acqui- 
sition was done using the pulse sequence in Figure 1A, using two selective gaussian pulses separated by a parametric delay $t_{1}$. To obtain the FID in Figure $2 \mathrm{~A}, 2065$ points were acquired in the $\mathrm{t}_{2}$ dimension and 96 in the $\mathrm{t}_{1}$ dimension. The spectra were processed using MatLab. A matched filter followed by Fast-Fourier-Transform and phasing were performed along the $t_{2}$ dimension. The point with the highest signal intensity was determined for each of the 96 points and is plotted as a function of $t_{1}$ in Figure 2A. The FID was zero-filled to 256 points then Fourier transformed as shown in Figure $2 \mathrm{C}$. The temperature during experiments was maintained at $20^{\circ} \mathrm{C}$ in order to maintain a constant exchange rate of the biosensor [10].

\section{Results and Discussion}

\subsection{Description of Biosensor Magnetization Transfer}

Magnetization transfer is commonly used with protons in chemical-exchangedependent saturation transfer, CEST, for signal enhancement and for enhancing imaging contrast [13]. Similarly, xenon has been used to provide signals from the gas-filled regions in lungs and to probe exchange times between different tissues in the lungs [8]. Although these techniques use exchange properties to transfer magnetization, they do not carry spectroscopic information while exchanging. Remote detection experiments have already shown that the long $\mathrm{T}_{1}$ of xenon enables the encoding of spectroscopic information as a modulation of the z-magnetization that can be carried from one physical site to another [14]. Both the exchange properties of the xenon biosensor and the long $T_{1}$ of xenon can be used to carry spectroscopic information from one exchange site to another.

In biosensor solutions, xenon exists in three environments with distinct chemical shifts: xenon in solution, xenon encapsulated in the cryptophane cage and xenon gas bubbles. The xenon gas is assumed to play no role in our experiment, but is useful to reference spectra. Of central importance is the xenon exchanging between the biosensor and the solution, which occurs with a time constant of $\sim 70 \mathrm{~ms}$ at $20^{\circ} \mathrm{C}[15]$. Xenon atoms that spend time within the cryptophane cage quickly return to the solution. This property can be used to transfer a frequency encoded value of magnetization on the xenon in the cage to the xenon in solution.

Figure 1A shows the pulse sequence we employ. Xenon is bubbled into the solution. Then the flow is stopped by pinching the tubing on both ends of the sample tube while waiting for residual bubbles to clear. The xenon inside the cage is then selectively excited with a $90_{\mathrm{x}}$ gaussian pulse, allowed to precess 
during a $t_{1}$ delay, and then is stored as z-magnetization by applying another selective $90_{x}$ pulse. These encoded spins then exchange back into the solution carrying the stored information and are replaced by fresh xenon during $\mathrm{d}_{\mathrm{ex}}$. By repeating this selective excitation, evolution, and storage multiple times $\left(N_{\text {multi }}\right)$ a significant amount of encoded xenon accumulates, thus modulating the xenon magnetization in solution according to the $t_{1}$ increment. A $90_{x}$ hard pulse and acquisition then provides a signal to read out the value of z-magnetization of the water-dissolved xenon peak, which corresponds to one point of an indirect FID. Because the xenon in solution is at relatively high concentration, only one scan is needed to detect its signal.

As $t_{1}$ is successively incremented the evolution of the biosensor magnetization is mapped out indirectly as seen in Figure $2 \mathrm{~A}$ for a $5.6 \mu \mathrm{M}$ sample. For this FID, 96 points were collected in the $t_{1}$ dimension with $t_{1}$ increments of 0.505 ms resulting in a resolution of $20.6 \mathrm{~Hz}$ and spectral width of $1980.2 \mathrm{~Hz}$ in the frequency domain. In Figure 2B, a linear baseline correction was applied to the FID in Figure 2A to correct for loss of signal due to xenon atoms exchanging out of the cage before they were encoded during $t_{1}$ evolutions that were on the order of the exchange time of the biosensor. Because $T_{1}$ $(\sim 500 \mathrm{~s})$ is much longer than the maximum $\mathrm{t}_{1}$ evolution $(\sim 50 \mathrm{~ms})$, loss of encoded signal due to $\mathrm{T}_{1}$ relaxation from the first to the last point is negligible. Upon Fourier transformation, the two peaks of the biosensor are clearly seen in Figure 2C. Because only one component of transverse magnetization was stored, we are not able to discern between positive and negative frequency after Fourier transforming. Only the negative frequency is shown in Figure 2C for clarity. If frequency distinction is desired, then quadrature detection can be used by collecting a second scan for each $t_{1}$ increment using a $90_{y}$ storage pulse.

\subsection{Sensitivity of Indirect Detection}

Detecting the biosensor signal indirectly inevitably adds a second dimension, which results in an increase of experiment time relative to direct detection experiments. However, when detecting low biosensor concentrations, even a direct experiment such as ESA would require significant signal averaging and long experiment times. Thus, a simple model was constructed to predict relative signal-to-noise for direct and indirect detection to predict the sensitivity of each experiment. The method of indirect detection is analogous to that of remote detection; therefore the sensitivity comparison introduced by Granwehr and Seeley [16] was implemented.

The relative integrals of the solution-dissolved xenon and cage-bound xenon of the spectrum of an $83 \mu \mathrm{M}$ sample were used to determine relative signal inten- 
sity values that could then be extrapolated to lower concentrations and used as input values for the model. We assume $60 \%$ biosensor cage occupancy of xenon atoms and complete excitation of those spins [12]. These spins all contribute to the accumulating signal. However, every cycle does not contribute the same amount of signal because the reservoir of polarized xenon in solution is being incrementally depleted after every encoding step leading to an incrementally lower cage-bound signal. We take this into account by subtracting the spins encoded from those in solution, leaving fewer spins available to exchange into the cage for subsequent encoding. This process can be written as a recursive relationship as shown in equation 1 for the signal in the indirect dimension

$$
\mathrm{S}_{\text {indirect }}=\sum_{n=1}^{N_{\text {multi }}}[\mathrm{bio}]\left(1-\frac{[\mathrm{bio}]}{[\mathrm{Xe}]_{0}}\right)^{n-1} \cdot e^{-\frac{t}{T_{1}}}
$$

where $[\mathrm{bio}]$ is the concentration of the biosensor, $[\mathrm{Xe}]_{0}$ is the initial concentration of the xenon in solution, $N_{\text {multi }}$ is the number of loops through the encoding/exchange part of the experiment, and $t$ is the time it takes experimentally to complete $N_{\text {multi }}$ loops. A similar recursive equation can be written for ESA with an additional term to account for the noise during the acquisition after each FID shown in equation 2.

$$
\mathrm{S}_{\mathrm{ESA}}=\sum_{n=1}^{N_{\text {multi }}}[\mathrm{bio}]\left(1-\frac{[\mathrm{bio}]}{[\mathrm{Xe}]_{0}}\right)^{n-1} \cdot \frac{e^{-\frac{t}{T_{1}}}}{\sqrt{n}}
$$

Plots of the $\mathrm{S} / \mathrm{N}$ for various biosensor concentrations are shown in Figures $3 \mathrm{~A}$ and $3 \mathrm{~B}$. These plots give the theoretical value of optimal $N_{\text {multi }}$ values for the direct and indirect experiments and the maximum signal that can be expected. As can be seen on both curves, $\mathrm{T}_{1}$ decreases the amount of signal during large $N_{\text {multi }}$ values. The $N_{\text {multi }}$ value that gives the maximum signal for our indirect method is determined from the plot; then a total experiment time can be calculated to achieve a desired $\mathrm{S} / \mathrm{N}$ for the experiment. To make a signal-to-noise-per-time comparison between our indirect detection and ESA, we calculated how many ESA experiments can be performed in the same time it takes to run an indirect experiment. The sensitivity comparison of the first point of both FIDs using one approach of Granwher and Seeley [16] is calculated according to equations 3,4 , and 5

$$
\begin{aligned}
& \Psi_{\mathrm{i}}=S_{\text {indirect }} \sqrt{\frac{T_{2}}{2 t_{\mathrm{i}}^{\text {rep }}}\left(1-\exp \left(-\frac{2 t_{\mathrm{i}}^{\mathrm{max}}}{T_{2}}\right)\right)} \\
& \Psi_{\mathrm{ESA}}=S_{\mathrm{ESA}} \sqrt{\frac{N_{t r} \cdot t_{\mathrm{ESA}}^{\mathrm{max}}}{t_{\mathrm{ESA}}^{\mathrm{rep}}}}
\end{aligned}
$$




$$
\text { relative sensitivity }=\frac{\Psi_{\mathrm{i}}}{\Psi_{\mathrm{ESA}}}
$$

where $t_{i}^{\max }$ and $t_{\mathrm{ESA}}^{\max }$ are the acquisition times of the indirect and direct experiments, respectively, $t_{i}^{\text {rep }}$ and $t_{\mathrm{ESA}}^{\text {rep }}$ are the repetition times of the two experiments (the time to acquire one point indirectly and one transient directly, respectively), and $N_{t r}$ is the number of direct transients that can be performed in the same amount of time as the indirect experiment. Relative sensitivity is plotted in Figure 4. As can be seen, using indirect detection becomes advantageous as the concentration is lowered, with the cross over point occurring at $6.7 \mu \mathrm{M}$.

The sensitivity of indirect detection is better appreciated experimentally in Figure 5. It shows the solution-dissolved xenon peak for the first 16 indirect points of a $1.1 \mu \mathrm{M}$ sample collected in $43 \mathrm{~min}$. A modulation on top of the peak can be clearly observed verifying the presence of biosensor whereas for an ESA experiment run for the same amount of time, no signal is observed. Therefore, indirect detection can give more information per unit time than ESA.

\subsection{Influence of Multiplicative Noise}

Although the theoretical results show that indirect detection has an advantage at lower concentrations, this advantage is difficult to experimentally realize because of multiplicative noise. Multiplicative noise arises because instabilities in the system affect the value of detected magnetization of each indirect point. In our flow setup the fluctuations in magnetization can be caused by the xenon polarizer (i.e. instability of the gas flow rate, variations in polarization, $\mathrm{T}_{1}$ relaxation within the manifold) or perturbations within the sample (i.e. motion from xenon gas bubbles or from the capillaries). These two effects are difficult to seperate but their collective contributions can account for up to $20 \%$ uncertainty in acquired signal. In principle these sources of noise can be reduced by technical improvements such as using a flow controller to prevent drifts in the flow or converting to a continuous-flow polarizer. The amplitude of multiplicative noise is an inherent limitation of the flow setup and not of the experiment itself. With additional improvements, the theoretical $\mathrm{S} / \mathrm{N}$ value is certainly approachable. 


\section{Conclusions}

We have shown previously that magnetization from xenon associated with biosensors is transferred to xenon in solution through chemical exchange. Here we demonstrate that this exchange can be exploited to carry and add modulated signals from the biosensor. Acquisition of this modulated signal as an indirect dimension in a 2D NMR experiment can provide the spectrum of the biosensor with enhanced sensitivity for dilute biosensors. Because the biosensor collects magnetization from many repeated frequency-label and exchange cycles before readout this approach has a gain of $\sqrt{N_{\text {multi }}}$ in $\mathrm{S} / \mathrm{N}$ relative to direct detection of the biosensor signal. The number of repeated cycles is limited by the relative concentration of the biosensor and dissolved xenon and by the $\mathrm{T}_{1}$ value of the dissolved xenon. However, for realistic concentrations of interest for detecting biomolecules, this approach should be advantageous when scan to scan variation (giving rise to ' $t_{1}$ noise') can be minimized. Finally, this technique demonstrates the feasibility of remote biosensor spectroscopy as discussed in reference [12]. With modifications to the flow setup, multiplicative noise can be minimized and encoded xenon gas can be extracted from the solution and carried to a more sensitive detector (small NMR coil, SQUID, or optical magnetometer), thus realizing true remote detection. Coupling exchange mediated magnetization transfer with remote detection would further increase sensitivity because encoding and detection could be separately optimized.

\section{References}

[1] T. G. Walker, W. Happer, Spin-exchange optical pumping of noble-gas nuclei, Rev. Mod. Phys. 69 (1997) 629-642.

[2] S. M. Rubin, M. M. Spence, I.E. Dimitrov, E. J. Ruiz, A. Pines, D. E. Wemmer, Detection of a conformational change in maltose binding protein by xe-129 NMR spectroscopy, J. Am. Chem. Soc. 123 (2001) 8616-8617.

[3] S.M. Rubin, M. M. Spence, A Pines, D. E. Wemmer, Characterization of the effects of nonspecific xenon-protein interactions on 129Xe chemical shifts in aqueous solutions: Further developmenment of xenon as a biomolecular probe, J. Magn. Reson. 152 (2001) 79-86.

[4] E. Locci, Y. Dehouck, M. Casu, G. Saba, A. Lai, M. Luhmer, J. Reisse, K. Bartik, Probing protein in solution by 129Xe NMR spectroscopy, J. Magn. Reson. 150 (2001) 167-174.

[5] T.J. Lowery, S.M. Rubin, E.J. Ruiz, A. Pines, S. E. Wemmer, Design of a conformation-sensitive xenon binding cavity in the ribose-binding protein, Angew Chem 43 (2004) 6320-6322 
[6] T. J. Lowery, M. Doucleff, E.J. Ruiz, S. M. Rubin, A. Pines, D. E. Wemmer, Distinguishing multiple chemotaxis $\mathrm{Y}$ protein conformations with laser-polarized Xe-129 NMR, Protein Science 14 (2005) 848-855.

[7] B. M. Goodson, Nuclear magnetic resonace of laser-polarized noble gases in molecules, materials and organisms, J. Magn. Reson. 155 (2002) 157-216.

[8] K. Ruppert, J.R. Brookeman, KD. Hagspiel, J.P. Mugler III, Probing lung physiology with xenon polarization transfer contrast (XTC), Magn. Reson. Med. 44 (2000) 349-357.

[9] M. M. Spence, S.M. Rubin, I.E. Dimitrov, E.J. Ruiz, D.E. Wemmer, A. Pines, S.Q. Yao, F. Tian, P.G. Schultz, Functionalized xenon as a biosensor, Proc. Natl. Acad. Sci. U.S.A. 98 (2001) 10654-10657.

[10] T. J. Lowery, S. Garcia, L. Chavez, E. J. Ruiz, T. Wu, T. Brotin, J. P. Dutasta, D. S. King, P. G. Schultz, A. Pines, D. E. Wemmer, Opimization of xenon biosensors for detection of protein interactions, ChemBioChem 7 (2006) 63-73.

[11] C. Hilty, T.J. Lowery, D.E. Wemmer, A. Pines, Spectrally resolved magnetic resonance imaging of a xenon biosensor, Angew. Chem. 45 (2006) 70-73.

[12] S. Han, S. Garcia, T.J. Lowery, E. J. Ruiz, J.A. Seeley, L. Chavez, D. S. King, D. E. Wemmer, A. Pines, NMR-Based biosensing with optimized delivery of polarized 129Xe to solutions, Anal. Chem. 77 (2005) 4008-4012.

[13] V. Guivel-Schare, T. Sinnwell, S.D. Wolff, R.S. Balaban, Detection of proton chemical exchange between Metabolites and water in biological tissues, J. of Magn. Reson. 133 (1998) 36-45.

[14] A.J. Moule, M.M. Spence, S. Han, J.A. Seeley, K. L. Pierce, S. Saxena, A. Pines, Amplification of xenon NMR and MRI by remote detection, Proc. Natl. Acad. Sci. U.S.A. 100 (2003) 9122-9127.

[15] M. M. Spence, E.J. Ruiz, S.M. Rubin, T.J. Lowery, N. Winssinger, P.G. Schultz, D. E. Wemmer, A. Pines, Development of a functionalized xenon biosensor, J. Am. Chem. Soc 126 (2004) 15287-15294.

[16] J. Granwehr, J.A. Seeley, Sensitivity quantification of remote detection NMR and MRI, J. of Magn. Reson. 179 (2006) 229-238. 


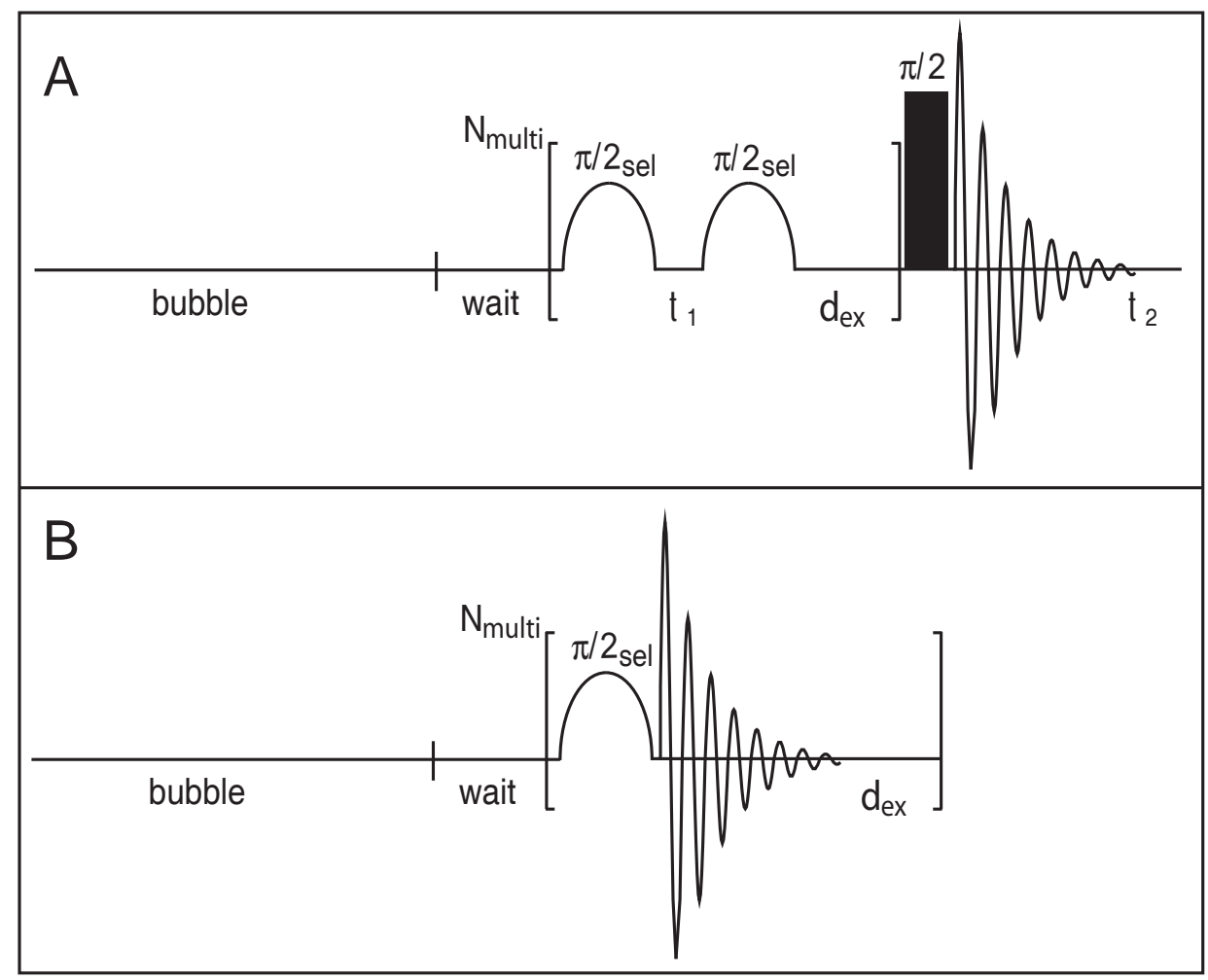

Fig. 1. (A) Timing of the pulse sequence used to transfer magnetization carrying spectroscopic information from one exchange site to another. Xenon flows into the sample for $25 \mathrm{~s}$ during bubble. A wait time of $10 \mathrm{~s}$ ensures that the bubbles have cleared before pulsing. A $90 \mathrm{x}$ gauss pulse $(1.25 \mathrm{~ms})$ corresponding to a $20 \mathrm{ppm}$ bandwidth excites the biosensor frequency followed by another gauss (also 90 ) pulse after $t_{1}$ evolution. The exchange delay was set to $300 \mathrm{~ms}$. The pulses and delays within the brackets can be repeated for multiple times $\left(N_{\text {multi }}\right)$ depending on the concentration. Finally, a $90_{\mathrm{x}}$ hard pulse is used to read off the magnetization value of the xenon dissolved in solution. The acquisition time, $t_{2}$, was set to 83 ms. (B) The ESA pulse sequence has the same bubble and wait times as indirect detection and similarly the components within the brackets can be repeated $N_{\text {multi }}$ times. 


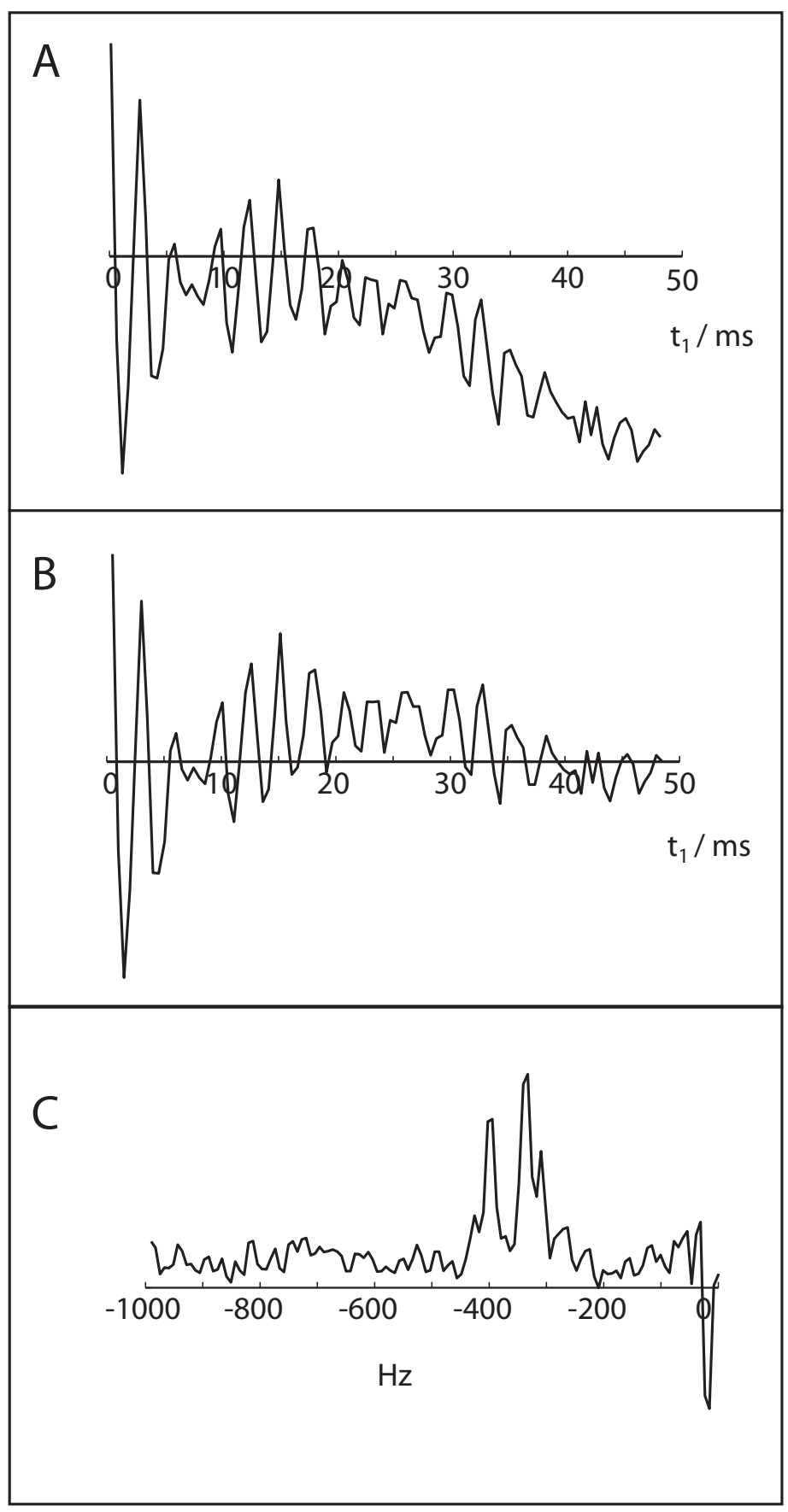

Fig. 2. The solution signal is modulated according to the biosensor signal for a 5.6 $\mu \mathrm{M}$ sample. (A) The FID is composed of 96 points each obtained from the maximum signal of the $t_{2}$ dimension. When the $t_{1}$ delay is on the order of the exchange time of the biosensor, signal is lost because xenon atoms exchange before the storage pulse. (B) A linear base line correction is applied to remove this effect. (C) Fourier transforming the FID yields the frequencies of the biosensor peaks. 

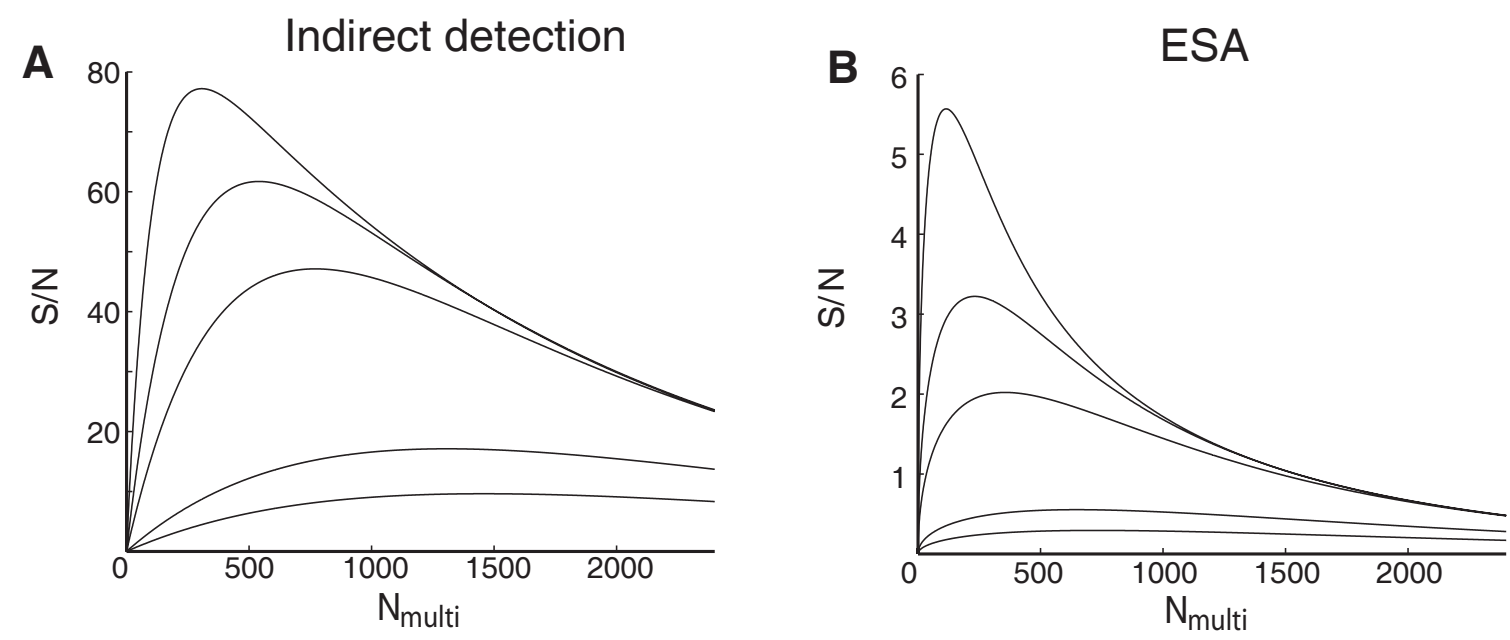

Fig. 3. Plots of theoretical signal for an indirect detection experiment (A) and one ESA experiment (B). The concentrations used were $0.5,1,5,10$ and $25 \mu \mathrm{M}$ with $0.5 \mu \mathrm{M}$ corresponding to the curve with the lowest signal and $25 \mu \mathrm{M}$ the highest. Note that the vertical scales and experiment times are different. 


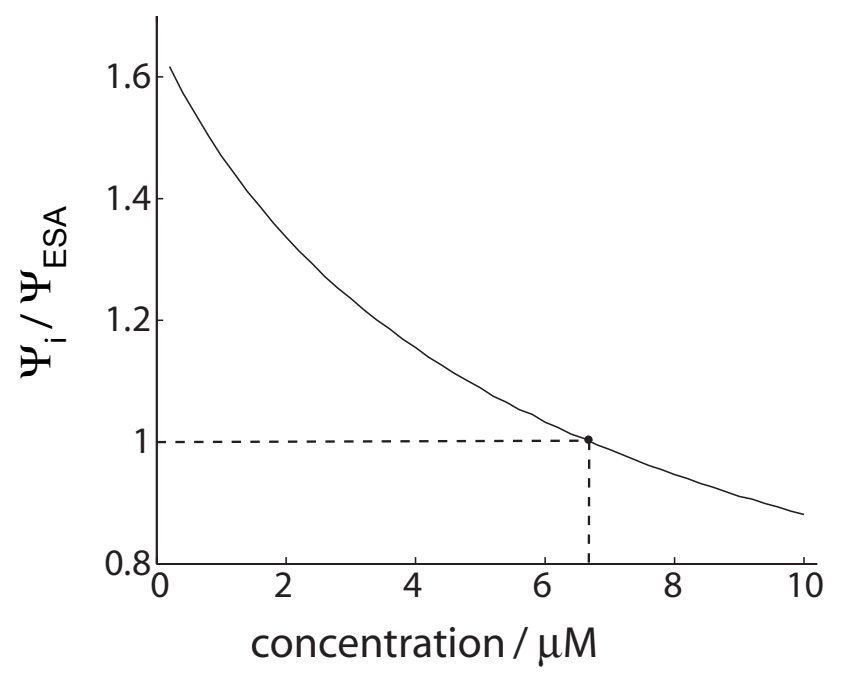

Fig. 4. Plot of the theoretical relative sensitivity between indirect detection and ESA as a function of biosensor concentration. Indirect detection is advantageous below a concentration of $6.7 \mu \mathrm{M}$. 

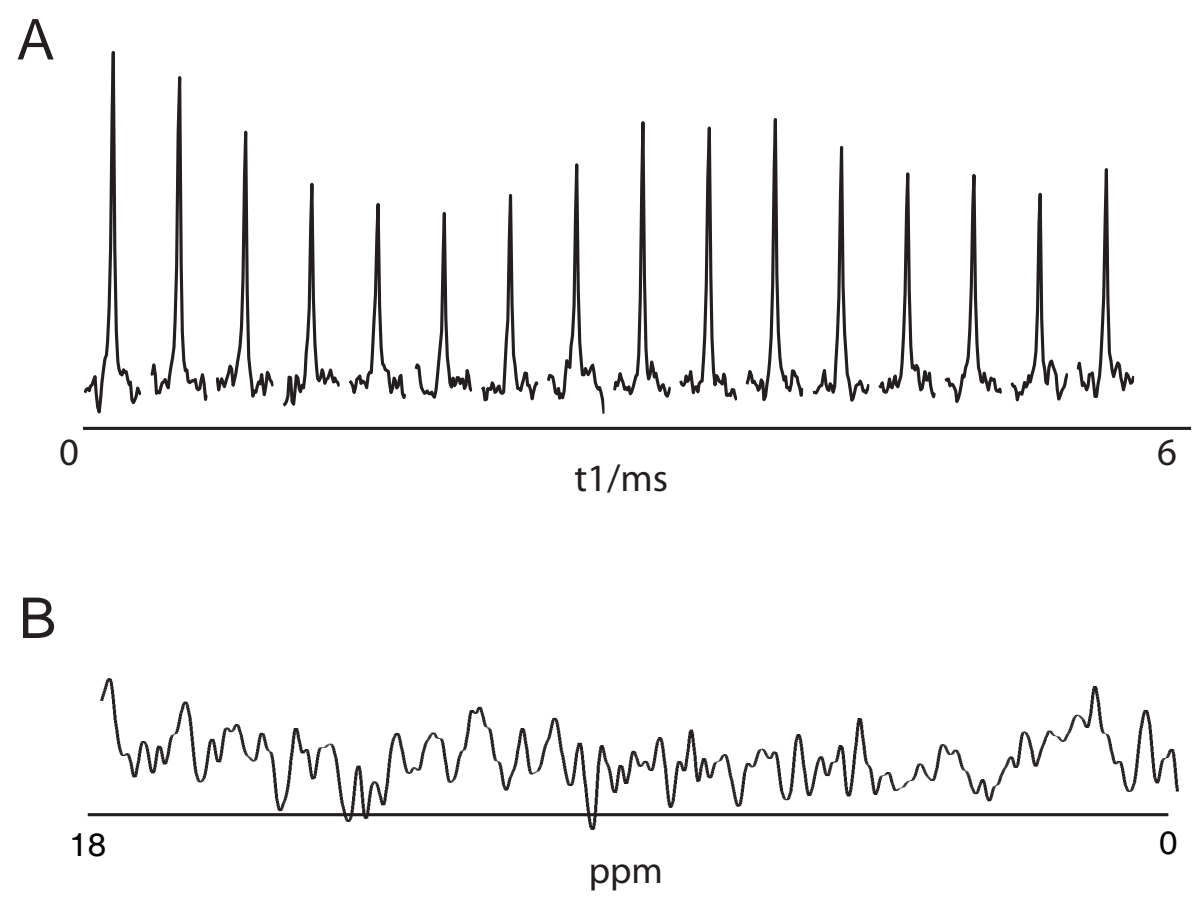

Fig. 5. A comparison between ESA and indirect detection was made at $1.1 \mu \mathrm{M}$ for a 43 min interval of experiment time, both having a $N_{\text {multi }}$ value of 450 . A modulation can be seen in the first 16 points of the indirect FID (A) but no signal is yet visible in the resulting 13 transients of the ESA spectrum (B). 